\title{
Quinolone-indolone conjugate induces apoptosis by inhibiting the EGFR-STAT3-HK2 pathway in human cancer cells
}

\author{
YING-HUA LIU, XIAO-LI WEI, GUO-QIANG HU and TIAN-XIAO WANG \\ Biopharmaceutical Department, Institute of Traditional Chinese Medicine, College of Pharmacy, \\ Henan University, Kaifeng, Henan 475004, P.R. China
}

Received July 11, 2014; Accepted March 26, 2015

DOI: $10.3892 / \mathrm{mmr} .2015 .3716$

\begin{abstract}
The epidermal growth factor receptor (EGFR) is involved in the proliferation of human tumors and is an effective target for the treatment of cancer. In the present study, a novel quinolone-indolone conjugate, QIC1 [9-Fluoro-3,7-dihydro-3-methyl-10- (4-methyl -1-piperazinyl) -6-(2-oxo-1,2-dihydro-indol-3-ylidenemethyl) -7-oxo-2H-(1,4) oxazino(2,3,4-ij)quinoline], which targeted EGFR, was synthesized in order to investigate the anticancer activity and the potential mechanisms underlying the effect of this compound in human cancer cells. Using MTT assays it was observed that QIC1 inhibited the growth of HepG2 human hepatoma cells, MCF7 human breast cancer cells, HeLa human cervical cancer cells and A549 human lung adenocarcinoma cells. QIC1 arrested cell cycle progression at the G2/M phase in HepG2 cells. QIC1 inhibited the synthesis of DNA in A549 cells. In addition, it resulted in cell apoptosis, in association with increased expression of Bax and reduced expression of Bcl-2. Further analyses demonstrated that QIC1 attenuated the activity of EGFR, and the downstream signal transducer and activator of transcription 3 (STAT3)-mediated hexokinase II (HK2) signaling pathways. Furthermore, QIC1 exhibited antiproliferative effects in MCF7/DOX human doxorubicin-resistant breast cancer cells and also enhanced the anticancer activity of doxorubicin in these cells. In conclusion, the inhibition of proliferation and the induction of apoptosis was associated with reduced expression of phospho-EGFR-phospho-STAT3-HK2. The present results suggest a potential role for QIC1 in the treatment of human cancer.
\end{abstract}

Correspondence to: Professor Tian-Xiao Wang, Biopharmaceutical Department, Institute of Traditional Chinese Medicine, College of Pharmacy, Henan University, Jinming Avenue, Kaifeng, Henan 475004, P.R. China

E-mail: wtx1975@126.com

Key words: quinolone-indolone conjugate, cancer, epidermal growth factor receptor, hexokinase II, signal transducer and activator of transcription 3

\section{Introduction}

Cancer remains a significant cause of morbidity and mortality (1), and there has been extensive research into the development of antitumor agents. In recent years, there has been an emergence of therapeutics directed against specific signaling pathways that are critical for the onset and progression of cancer.

Protein tyrosine kinases are involved in processes, such as cell proliferation, survival, apoptosis, migration, and DNA damage repair, and have therefore received attention as potential novel anticancer agents. The epidermal growth factor receptor (EGFR), which is a transmembrane receptor tyrosine kinase, is involved in the proliferation of human tumors and is an effective target for the treatment of cancer (2-5). The present study aimed to identify novel potent indolin-2-one protein tyrosine kinase inhibitors; indolin-2-one derivatives were synthesized using sunitinib as a leading compound. A quinolone-indolone conjugate QIC1 [9-Fluoro-3,7-dihydro-3-methyl-10- (4-methy 1-1-piperazinyl)-6-(2-oxo-1,2-dihydro-indol-3-ylidenemethyl) -7-oxo-2H-(1,4) oxazino(2,3,4-ij)quinoline], which targeted EGFR, was synthesized.

The EGFR pathway comprises a number of downstream signal transduction cascades, such as extracellular signal-regulated kinase $1 / 2$, protein kinase B (AKT) and signal transducer and activator or transcription 3 (STAT3). Inhibition of EGFR leads to downregulation of these signaling cascades, resulting in the apoptosis of cancer cells (6). STAT3 is overexpressed and constitutively activated in a number of types of cancer cells. Blocking STAT3 signaling inhibits cell growth, induces apoptosis and reduces tumor cell metastasis (7,8). STAT3 regulates the expression of numerous genes, such as CyclinD1, Bcl-2, Bax, Survivin, as well as other genes that regulate cell cycle progression and cell proliferation $(9,10)$. STAT3 is proposed to be one of the most important oncoproteins and a potential target for cancer therapy $(11,12)$. Within cancer cells, a particular metabolic pathway, which is characterized by the anaerobic degradation of glucose even in the presence of oxygen, leads to the production of large quantities of lactate; a phenomenon known as the Warburg effect $(13,14)$. Clinical studies have confirmed that enhanced glucose degradation occurs within tumors (15-17). Hexokinase II (HK2), which catalyzes the first committed step of glycolysis, is overexpressed and activated in a number of cancers. Therefore HK2 
has also been investigated as a target of cancer therapy (18). Furthermore, previous studies by this group have demonstrated that STAT3 may regulate the expression of HK2 (19-21).

Therefore, it was hypothesized that EGFR, STAT3 and HK 2 constitute a pathway that regulates cancer progression. The present study investigated the activity of QIC1 against liver, lung, cervix and breast cancer cells, in addition to the mechanisms underlying the effect of QIC1 on the EGFR pathway, which result in apoptosis in cancer cells.

\section{Materials and methods}

Cell lines, antibodies and reagents. MCF7 (human breast cancer), HepG2 (human hepatocellular carcinoma), A549 (human lung cancer), HeLa (human cervical cancer) cells and QSG7701 healthy human hepatocytes were procured from the American Type Culture Collection (Manassas, VA, USA). The following antibodies were obtained from Cell Signaling Technology, Inc., (Danvers, MA, USA): Mouse monoclonal anti-phosphorylated EGFR (pTyr1068-EGFR) (1:1,000; cat. no. 2236), rabbit polyclonal anti-STAT3 (1:1,000; cat. no. 9132), rabbit polyclonal anti-phosphorylated STAT3 (pTyr705-STAT3) (1:1,000; cat. no. 9131), rabbit monoclonal anti-AKT (1:1,000; cat. no. 4685), rabbit monoclonal anti-phosphorylated AKT (pSer473-AKT) (1:1,000; cat.no. 4060), rabbit monoclonal anti-Bax (1:1,000; cat. no. 5023), rabbit monoclonal Bcl-2 (1:1,000; cat. no. 2870) and rabbit monoclonal HK-2 (1:1,000; cat. no. 2867). Rabbit polyclonal anti-EGFR (1:1,000; cat. no. 18986-1-AP), rabbit polyclonal anti- $\beta$-actin (1:2,000; cat. no. 20536-1-AP) primary antibodies and peroxidase-conjugated Affinipure goat anti-rabbit/mouse secondary antibodies (1:10,000; cat. nos. SA00001-2 and SA00001-1) were obtained from Proteintech Group, Inc. (Chicago, IL, USA). EasyScript Two-Step RT-PCR SuperMix kit was obtained from TransGen Biotech (Beijing, China). Propidium iodide (PI), RNase A and Hoechst 33342 were obtained from Sigma-Aldrich (St. Louis, MO, USA). Triton X-100 was obtained from Amresco LLC (Solon, OH, USA). TRIzol was obtained from Biyuntian biotech (Shanghai, China). Agarose was purchased from Invitrogen Life Technologies (Carlsbad, CA, USA).

Effect of STAT3 small interfering (si)RNA and HK-2 inhibitor on anticancer activity of QICl. HepG2 cells were transfected with STAT3 siRNA or treated with the HK-2 inhibitor, 3-BrPA for $6 \mathrm{~h}$. Subsequently, different concentrations of QIC1 $(0,1$, 2 and $4 \mu \mathrm{M})$ was added. After $48 \mathrm{~h}$, an 3-(4,5-dimethylthiazol-2-yl)-2,5-diphenyl-tetrazolium bromide (MTT) assay was performed to detect the cell viability to evaluate whether QIC1 regulates cancer cell growth through the STAT3-HK2 pathway.

Cell culture and cell viability assay. Cells were cultured in RPMI-1640 (Invitrogen Life Technologies) supplemented with $10 \%$ fetal bovine serum and $1 \%$ penicillin/streptomycin in $5 \%$ $\mathrm{CO}_{2}$ at $37^{\circ} \mathrm{C}$. The quinolone-indolone conjugate, QIC1, was synthesized chemically by aldol condensation of the quinolone $\mathrm{C}-3$ position, in order to introduce indolin-2-one. QIC1 was dissolved in DMSO (Sigma-Aldrich). An MTT assay was used to detect cell viability and cytotoxicity. MCF7, HepG2,
HeLa, A549 and QSG7701 cells, cultured in 96-well plates, were treated with final concentrations of $0.5,1,2$ and $4 \mu \mathrm{M}$ of QIC1 for $48 \mathrm{~h}$. Treated cells were then incubated in fresh medium containing MTT $\left(0.5 \mathrm{mg} / \mathrm{ml}\right.$; Sigma-Aldrich) at $37^{\circ} \mathrm{C}$ for $4 \mathrm{~h}$ followed by replacement with $150 \mu \mathrm{l}$ DMSO. Finally, the spectrophotometric absorbance of the samples in DMSO was determined using a microplate reader (Sunrise ${ }^{\mathrm{TM}}$, Tecan Group Ltd., Männedorf, Switzerland) at $570 \mathrm{~nm}$.

Cell cycle analysis. Cell cycle analysis was performed using flow cytometry. HepG2 and A549 cells treated with QIC1 $(0,1,2$ or $4 \mu \mathrm{M})$ for $48 \mathrm{~h}$ were collected and washed twice with ice-cold PBS. Cells $\left(1 \times 10^{6}\right)$ were fixed in $75 \%$ ethanol at $4^{\circ} \mathrm{C}$ for $\geq 4 \mathrm{~h}$. Cells were then washed twice with ice-cold PBS, followed by incubation with DNA staining solution [(PI; $50 \mu \mathrm{g} / \mathrm{ml})$, RNase $(50 \mu \mathrm{g} / \mathrm{ml})$ and Triton-X-100 (0.5\%)] for $20 \mathrm{~min}$. Cell cycle analysis was immediately performed using flow cytometry (FACSCalibur, BD Biosciences, Franklin Lakes, NJ, USA).

Cell apoptosis analysis. Cell apoptosis was analyzed using an ArrayScan VTI HCS 600-type high content live cell imaging system (Thermo Fisher Scientific, Waltham, MA, USA). MCF7, HepG2 and A549 cells were seeded in 96-well plates. After $12 \mathrm{~h}$, the cells were treated with QIC1 $(0,0.5,1,2$ and $4 \mu \mathrm{M})$ for $48 \mathrm{~h}$, and then washed with ice-cold PBS. Cells were incubated with $5 \mathrm{mg} / \mathrm{ml}$ Hoechst 33342 for $30 \mathrm{~min}$, and then incubated with $5 \mathrm{mg} / \mathrm{ml}$ PI for a further $1 \mathrm{~h}$ at $37^{\circ} \mathrm{C}$. The cells were subsequently washed with ice-cold PBS. The cell apoptosis analysis was immediately performed using the ArrayScan VTI HCS 600-type high content live cell imaging system. The second channel overall average fluorescence intensity (mean fluorescence intensity; MFI) represents cells in late apoptosis.

Western blotting. Cells treated with QIC1 were harvested in RIPA buffer (50 mM Tris- $\mathrm{HCl}, \mathrm{pH} 8.0 ; 150 \mathrm{mM}$ sodium chloride; $1.0 \% \mathrm{NP}-40 ; 0.5 \%$ sodium deoxycholate; and $0.1 \% \mathrm{SDS}$ ) with $10 \mu \mathrm{g} / \mathrm{ml}$ of the protease inhibitor, PMSF (Sigma-Aldrich). Total cellular proteins (40 $\mu \mathrm{g}$ ) were separated using SDS-PAGE and subjected to western blotting using primary antibodies against EGFR, pTyr1068EGFR, STAT3, pTyr705-STAT3, AKt, pSer473-AKT, HK, Bcl-2, Bax and $\beta$-actin (Cell Signaling Technology, Inc. and Proteintech Group, Inc.). $\beta$-actin was used as loading control. Bands were detected using an ECL detection system (AlphaImager; Proteinsimple, San Jose, CA, USA).

Reverse transcription-polymerase chain reaction (RT-PCR) analysis. Total mRNA was extracted from cultured cells using TRIzol. Reverse transcription and PCR reactions were performed using an Easy Script Two-Step RT-PCR SuperMix kit (TransGen Biotech company, Beijing, China). The primer pairs for RT-PCR were as follows: Forward: 5'-ATG GTCAAGTGCTGGATG-3' and reverse: 5'-GAGGAA GGTGTCGTCTATG-3' for EGFR, forward: 5'-CCA AGGAGGAGGCATTCG-3' and reverse: 5'-ACATCGGCA GGTCAATGG-3' for STAT3 and forward: 5'-GGTGCTGAG TATGTCATGGA-3' and reverse: 5'-TTCAGCTCTGGGAT GACCTT-3' for GAPDH (both sets from Sangon Biotech Co., Ltd., Shanghai, China). The PCR conditions were as follows: 
A<smiles>CC1COc2c(N3CCN(C)CC3)c(F)cc3c(=O)c(/C=C4\C(=O)Nc5ccccc54)cn1c23</smiles>

C

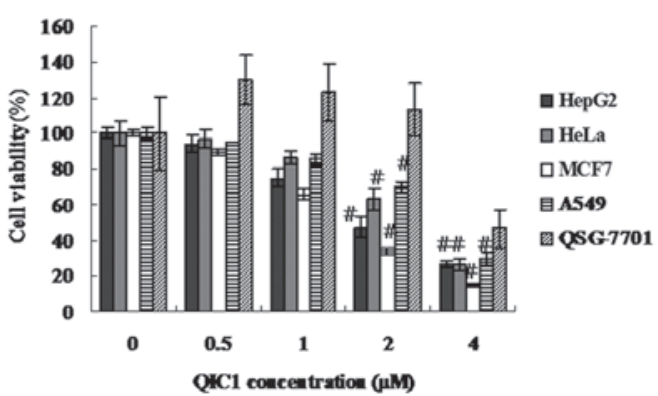

B<smiles>CCN(CC)CCNC(=O)c1c(C)[nH]c(/C=C2/C(=O)Nc3ccc(F)cc32)c1C</smiles>

D

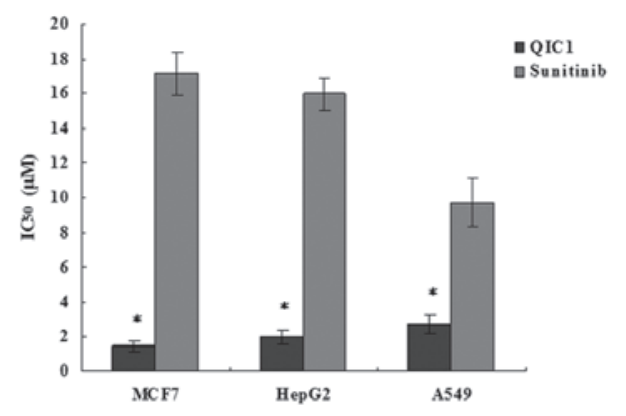

Figure 1. Antiproliferative effects of QIC1. (A) Schematic diagram of QIC1. (B) Schematic diagram of the positive control, sunitinib. (C) Antiproliferative effects of QIC1 were measured in various cancer cell lines, including HepG2, HeLa, MCF7 and A549 cells, as well as in QSG7701 normal human liver cells. Cells were exposed to QIC1 for $48 \mathrm{~h}$ prior to the MTT assay. Data are presented as the mean percentage \pm standard deviation, obtained from three independent experiments. (D) $\mathrm{IC}_{50}$ of QIC1 and Sunitinib in cancer cells. ${ }^{*} \mathrm{P}<0.05$ vs. no $\mathrm{QIC1}$ group, ${ }^{*} \mathrm{P}<0.01$ vs. positive control sunitinib. QIC1, quinolone-indolone conjugate.
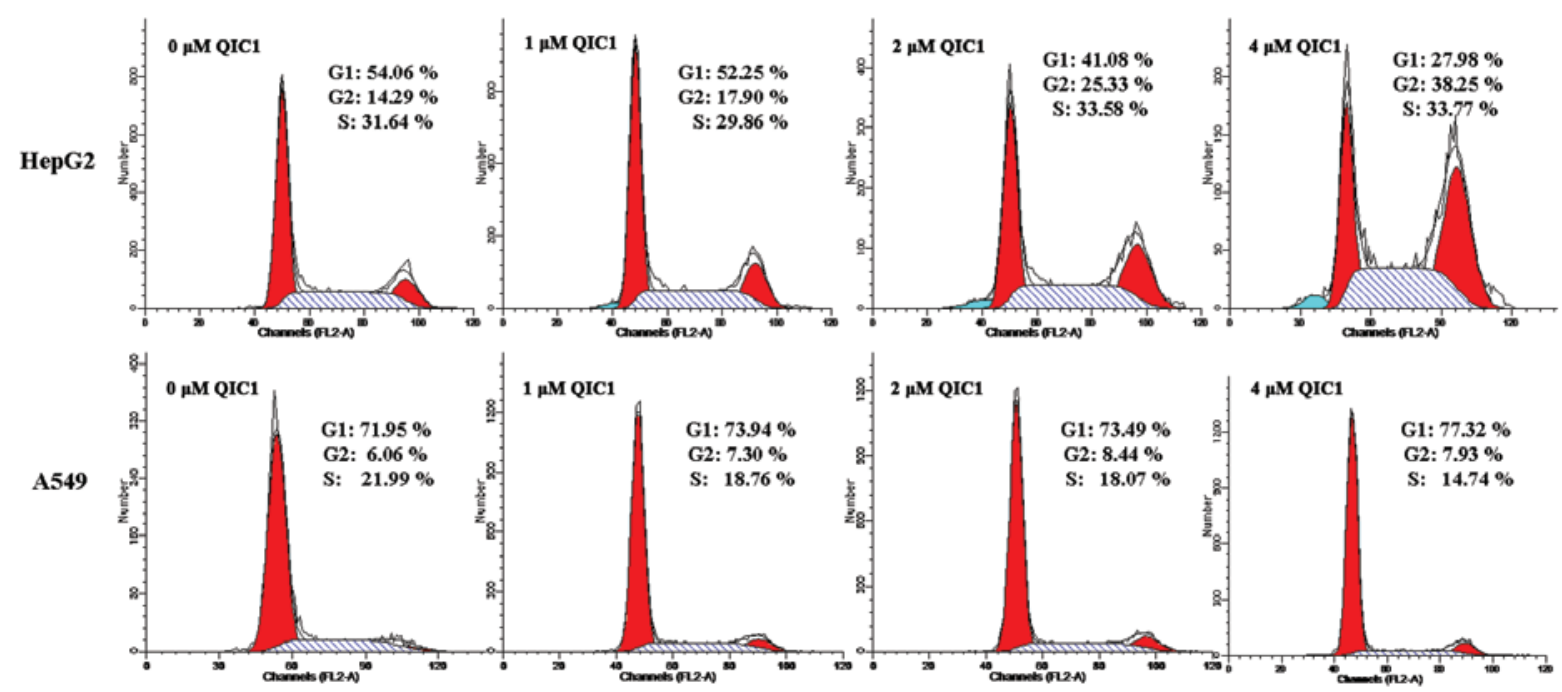

Figure 2. Cell cycle analysis. Cells were treated with QIC1 $(0,1,2$ or $4 \mu \mathrm{M})$ for $48 \mathrm{~h}$. Graphs show the percentage distribution of cells in each cell cycle phase. QIC1, quinolone-indolone conjugate.

$94^{\circ} \mathrm{C}$ for $5 \mathrm{~min}$, followed by 38 cycles at $94^{\circ} \mathrm{C}$ for $30 \mathrm{sec}, 56^{\circ} \mathrm{C}$ for $30 \mathrm{sec}$ and $72^{\circ} \mathrm{C}$ for $1 \mathrm{~min}$, an $72^{\circ} \mathrm{C}$ for 7 min using an Arktik PCR system (Thermo Scientific, Waltham, MA, USA). mRNA expression of GAPDH, EGFR and STAT3 was determined with density scanning (AlphaImager; Proteinsimple, San Jose, CA, USA). Subsequently the EGFR:GAPDH ratio and the STAT3:GAPDH (mRNAs were detected in the same RT sample) in densitometric units was calculated and analyzed using SPSS 17.0 software (SPSS Inc., Chicago, IL, USA).

Statistical analysis. Each analysis was repeated three times. Relative cell viability is expressed as a percentage relative to the untreated control cells. Error bars represent standard deviation. Data were analyzed using analysis of variance for each two-group comparison test. Statistical analyses were conducted using SPSS 17.0. $\mathrm{P}<0.05$ was considered to indicate a statistically significant difference.

\section{Results}

QIC1 inhibits the proliferation of cancer cells and is a non-cytotoxic compound. The quinolone-indolone conjugate, QIC1, was synthesized to target EGFR. It was hypothesized that QIC1 may target human cancers via its effect on EGFR. The schematic structural diagram of QIC1 is shown in Fig. 1A. The antiproliferative/survival activity of QIC1 in 
$\mathbf{A}$

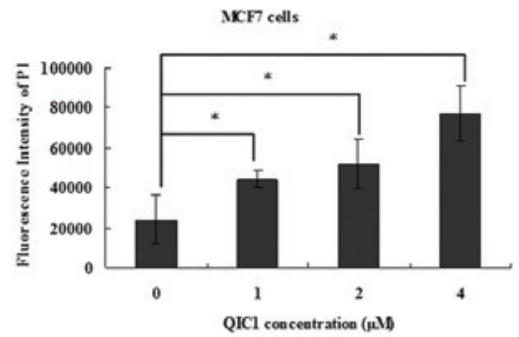

$\mathbf{B}$
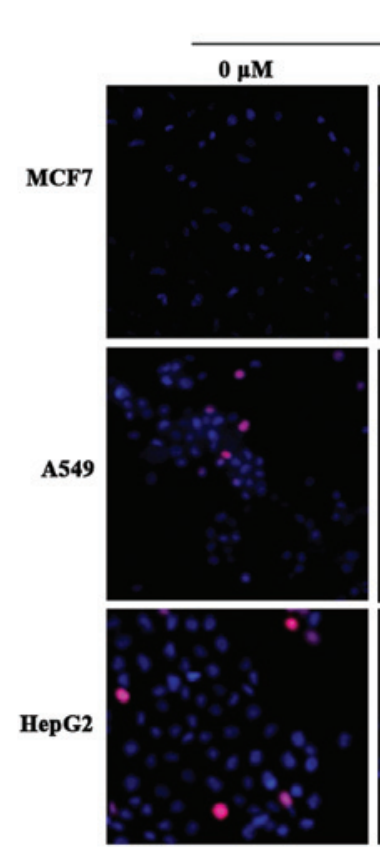

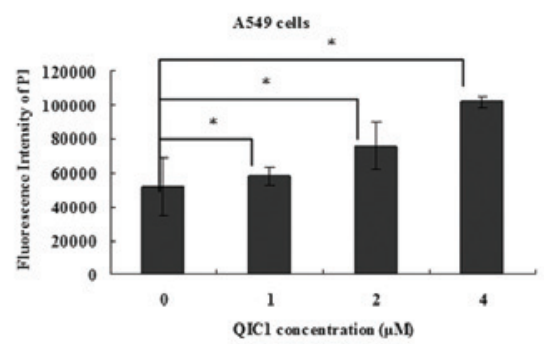

QIC1
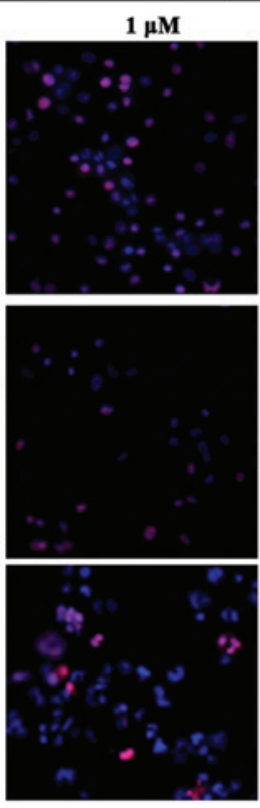
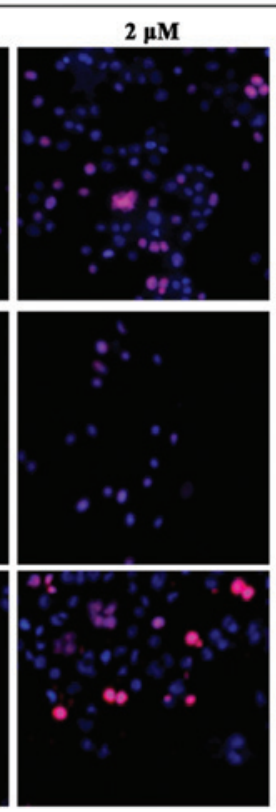
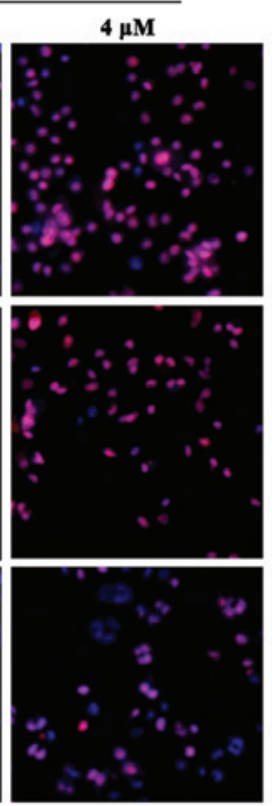

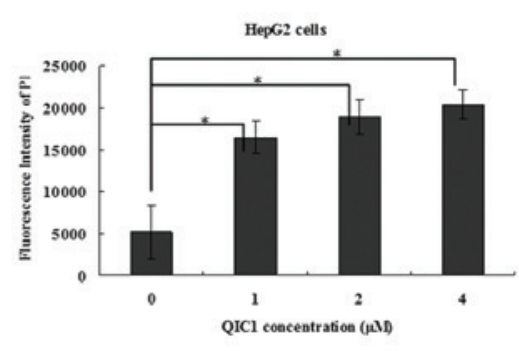

C
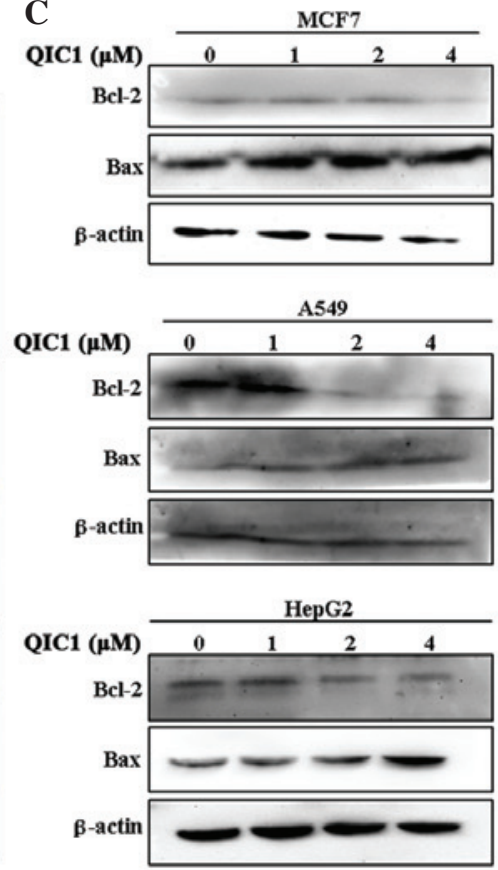

Figure 3. Induction of apoptosis in QIC1-treated cancer cells. (A) Effect of QIC1 on the fluorescence intensity of PI in cancer cells. Cells treated with 0, 1, 2 and $4 \mu \mathrm{M}$ of QIC1 for $48 \mathrm{~h}$ were incubated with $5 \mathrm{mg} / 1$ Hoechst 33342 for $10 \mathrm{~min}$ and $5 \mathrm{mg} / 1 \mathrm{PI}$ for a further $1 \mathrm{~h}$ in darkness. The MFI of PI was subsequently detected by an ArrayScan VTI HCS 600-type high content live cell imaging system. (B) Image of PI and Hoechst 33342 double-staining in different type of cancer cells. (C) Measurement of the effect of QIC1 on the expression of Bcl-2 and Bax by western blotting. "P<0.05. PI, propidium iodide; MFI, mean fluorescence intensity; QIC1, quinolon-indolone conjugate.

liver, lung, cervix and breast cancer cells was investigated and compared with that of the tyrosine kinase inhibitor, sunitinib (schematic structural diagram shown in Fig. 1B), which was used as a positive control. The activity of QIC1 was examined in MCF7, HepG2, HeLa and A549 cells, and QSG7701 healthy human hepatocytes, using an MTT assay. Among these cell types, QIC1 induced a significant dose-dependent decrease in cancer cell survival (Fig. 1C). In MCF7, HepG2, HeLa and A549 cells, the dose of QIC1 required to achieve $50 \%$ cell viability $\left(\mathrm{IC}_{50}\right)$ was $1.467,1.994$, 2.513 and $2.708 \mu \mathrm{M}$ respectively. Furthermore, QIC1 was shown to be a non-cytotoxic compound as QIC1 $(0.5,1$ and $2 \mu \mathrm{M})$ did not inhibit cell growth in the healthy QSG7701 hepatocyte cell line (Fig. 1C). QIC1 exhibited a stronger anticancer activity than that of sunitinib (Fig. 1D).

The effects of $\mathrm{QIC1}$ on cell proliferation were also examined using cell cycle analysis. There was a significant accumulation of the G2/M (4N-DNA) cell population in HepG2 cells treated with QIC1 (17.90, 25.33 and 38.25\% following incubation with 1,2 and $4 \mu \mathrm{M}$, respectively) compared with untreated cells (14.29\%; Fig. 2). In A549 cells, the S phase cell population was decreased, which indicated that QIC1 may inhibit DNA synthesis in A549 (Fig. 2). These observations suggest that
QIC1 may be a promising candidate with which to inhibit cancer cell survival and proliferation.

QIC1 induces cancer cell apoptosis. In subsequent experiments, the hypothesis that QIC1 results in cancer cell death by promoting apoptosis was investigated. Cell apoptosis was detected using an ArrayScan VTI HCS 600-type high content live cell imaging system. MCF7, HepG2 and A549 cells underwent apoptotic death in a dose-dependent manner when they were treated with QIC1 at doses of 1,2 and $4 \mu \mathrm{M}$ for $48 \mathrm{~h}$ (Fig. 3A and B). Furthermore, enhanced Bax expression and reduced Bcl-2 expression was observed in HepG2, MCF7 and A549 cells that were treated with QIC1 (1, 2 or $4 \mu \mathrm{M}$; Fig. 3C). These results supported the hypothesis of the induction of an apoptotic response in MCF7, HepG2 and A549 cancer cells upon treatment with QIC1.

QIC1 inhibits the EGFR-STAT3-HK2 pathway. Previous studies have indicated that downregulation of either the expression or the activity of EGFR contributes to the inhibition of cell proliferation and the induction of apoptosis in cancer cells $(22,23)$. In the present study, the effect of QIC1 on the expression and activity of EGFR was measured in 
A

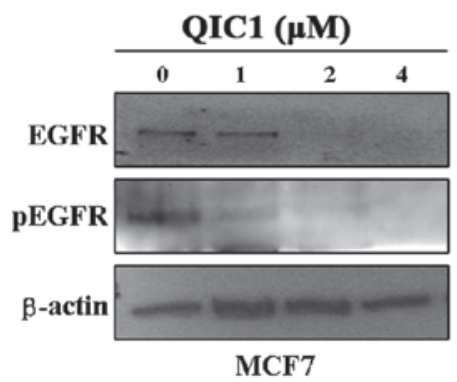

B

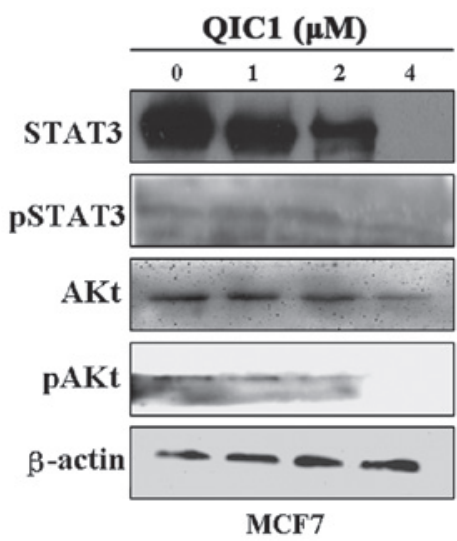

C

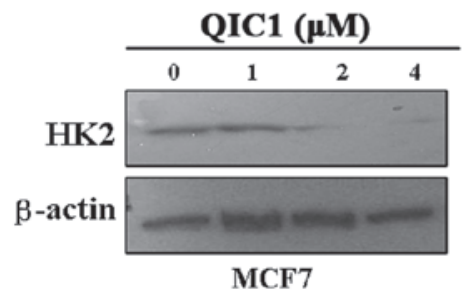

D

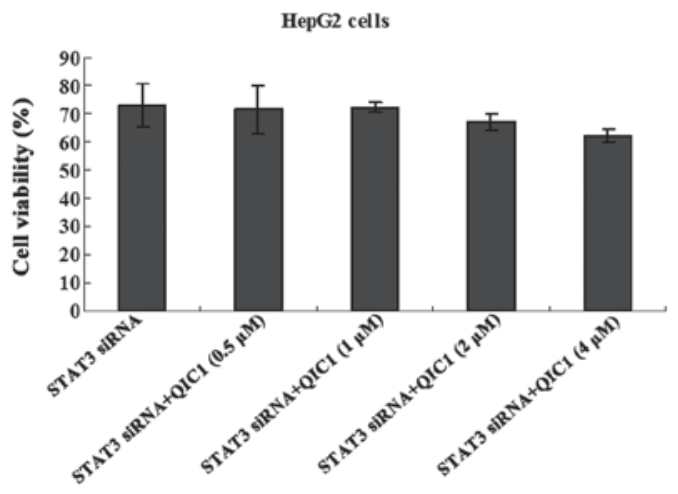

E

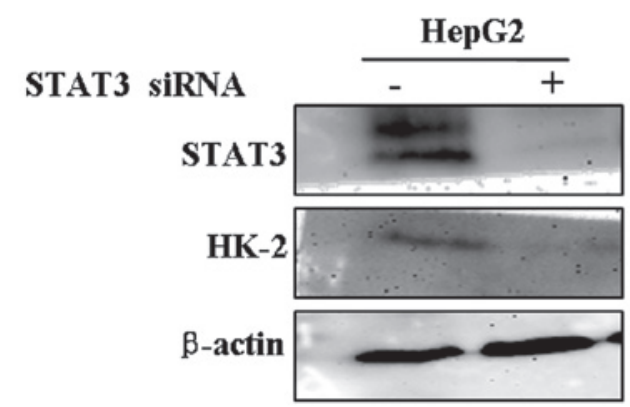

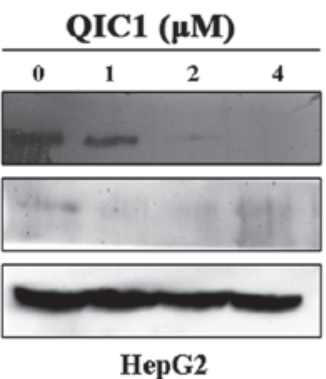

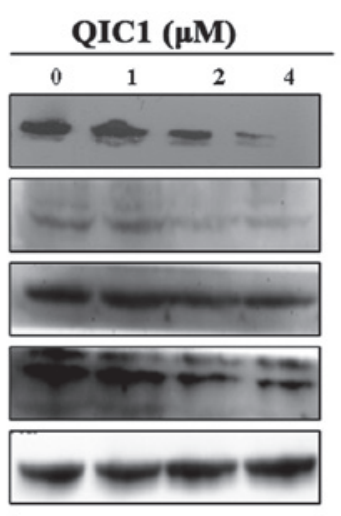

HepG2

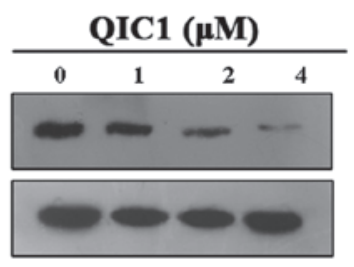

HepG2
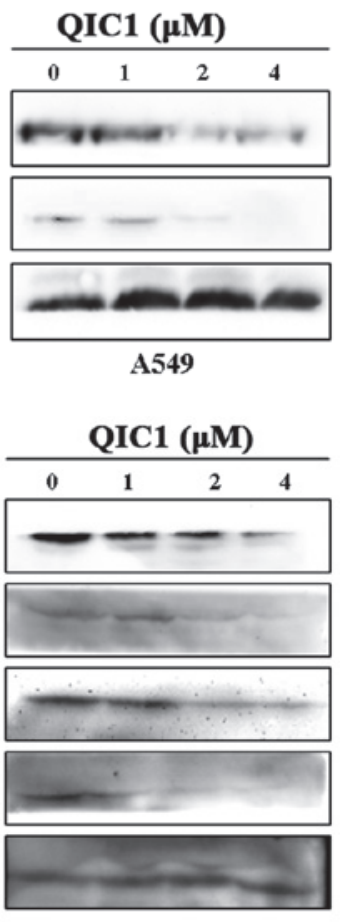

A549

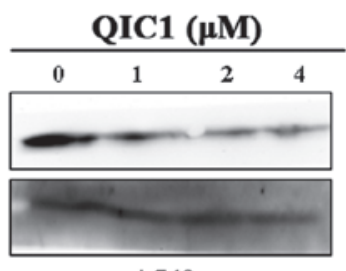

A549
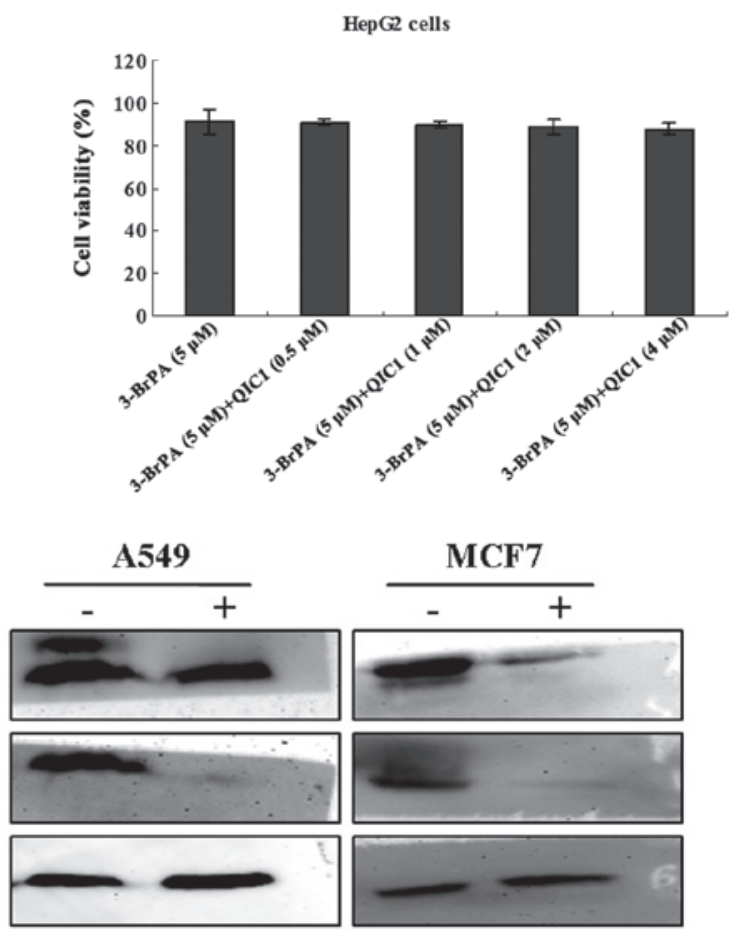

Figure 4. Evaluation of the inhibitory effect of QIC1 on the EGFR-STAT3-HK2 pathway. (A) Changes in EGFR and Phospho-EGFR levels were examined in MCF7, HepG2 and A549 cells following QIC1 treatment for $48 \mathrm{~h}$ by western blotting. (B) Whole cell lysates were prepared from cells treated with QIC1 for $48 \mathrm{~h}$ and immunoblotted to assess normal and activated forms of STAT3 and AKT. (C) Changes in the level of HK2 in QIC1-treated MCF7, HepG2 and A549 cells for $48 \mathrm{~h}$ were examined by western blotting. (D) The anticancer activity of QIC1 was counteracted by STAT3 siRNA and the HK2 inhibitor, 3-BrPA, using an MTT assay. (E) STAT3 siRNA. Changes in the expression of HK2 in MCF7, HepG2 and A549 cells following treatment with STAT3 siRNA for $48 \mathrm{~h}$ were detected by western blotting. QIC1, quinolone-indolone conjugate; EGFR, epidermal growth factor receptor; STAT3, signal transducer and activator of transcription 3; HK2, hexokinase II; AKT, protein kinase B; 3-BrPA, 3-bromine pyruvate; siRNA, small interfering RNA. 
A

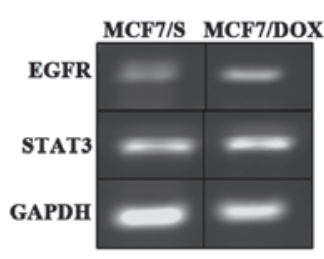

B

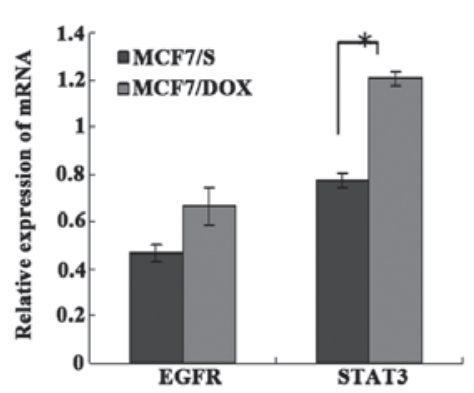

C

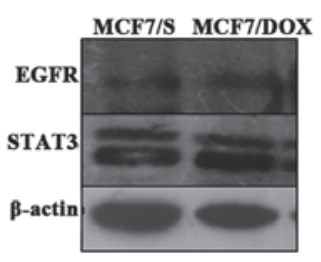

D

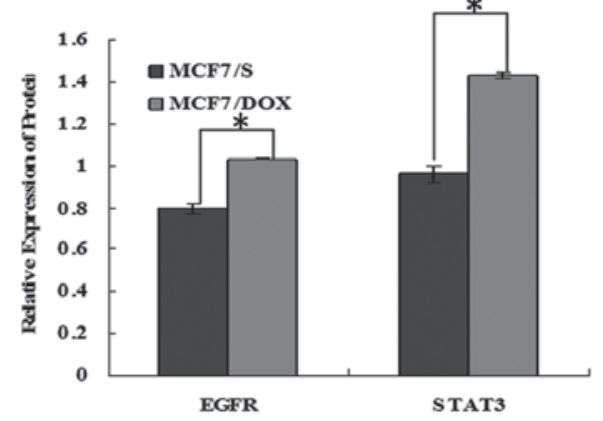

F

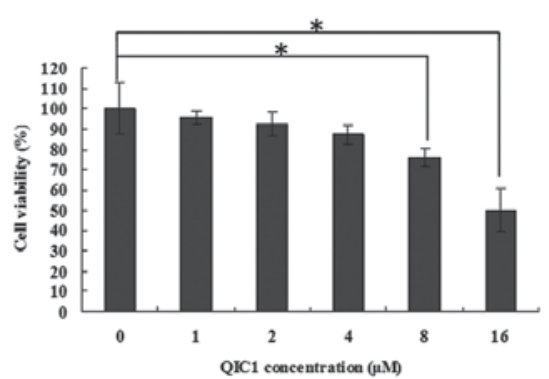

E

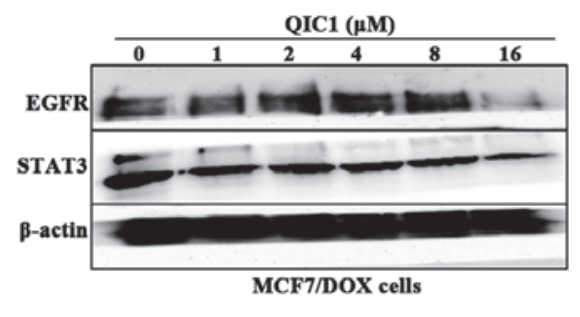

G

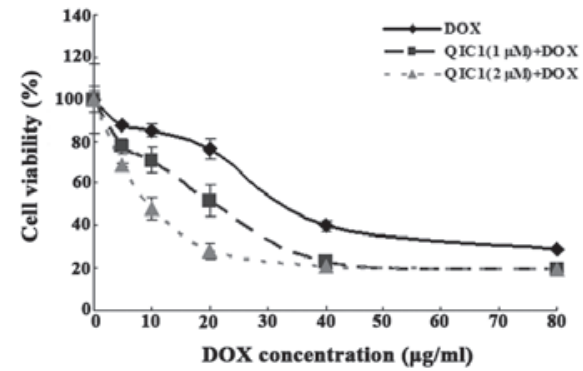

Figure 5. Involvement of QIC1 in doxorubicin-resistant MCF7/DOX human breast cancer cells. (A) and (B) mRNA levels of EGFR and STAT3 in MCF7/DOX and MCF7/S cells. (C) and (D) Protein levels of EGFR and STAT3 in MCF7/DOX and MCF7/S cells. (E) QIC1 inhibited the expression of EGFR and STAT3 in MCF7/DOX cells. (F) Antiproliferative effects of QIC1 in MCF7/DOX cells. Data are presented as the mean percentage \pm standard deviation obtained from three independent experiments. (G) QIC1 enhanced doxorubicin anticancer activity in MCF7/DOX cells. "P<0.05. QIC1, quinolone-indolone conjugate; EGFR, epidermal growth factor receptor; STAT3, signal transducer and activator of transcription 3.

MCF7, HepG2 and A549 cell lines. Notably, decreased EGFR activity and expression was observed in all three cell lines exposed to QIC1, in a dose-dependent manner (Fig. 4A). It has also been reported that certain downstream components of the EGFR signaling pathway, such as activated AKT and STAT3, are involved in cell survival and apoptosis (24-26). Therefore, the expression and activation status of these downstream signaling molecules was also investigated. Reduced expression and activity of AKt and STAT3 was observed in the three cancer cells treated with QIC1 (Fig. 4B). Previous studies by this group, have indicated that STAT3 may regulate the expression of HK2 in HepG2 cells. HK2 catalyzes the first committed step of glycolysis, an important metabolic pathway in cancer cells, which provides essential energy for the growth and proliferation of cancer cells. It has been shown to be overexpressed and activated in a number of cancers. The downregulation of HK2 inhibits the growth and proliferation of cancer cells. Therefore, the present study examined the effect of QIC1 on the expression status of HK2 in MCF7, HepG2 and A549 cell lines. Notably, decreased HK2 expression was observed in the cell lines exposed to QIC1, and this decrease occurred in a dose-dependent manner (Fig. 4C). In order to further confirm whether QIC1 regulates cancer cell growth through the EGFR-STAT3-HK2 pathway, cell growth status was measured after cancer cells were pretreated with STAT3 siRNA: Sense: 5'-GCAACAGAUUGCCUGCAUUdTdT-3' and antisense: 5'-AAUGCAGGCAAUCUGCAUU-3'), and the HK2 inhibitor, 3-bromine pyruvate (3-BrPA; Adamas-Beta, Shanghai, China). IT was observed that the anticancer activity of QIC1 was counteracted by the administration of STAT3 siRNA and 3-BrPA (Fig. 4D). Furthermore, STAT3 siRNA decreased the expression of HK2 in these cells (Fig. 4E). These results indicated that the downregulation of the EGFR-STAT3-HK2 pathway may be one if the mechanisms underlying the effect of QIC1.

QIC1 reverses drug resistance in human breast cancer MCF7/DOX cells. The results suggested that QIC1 may downregulate the expression and activity of EGFR and STAT3. Furthermore, a previous study demonstrated that the overexpression of STAT3 induces multidrug resistant in cancer cells and consequently, that STAT3 may be an effective 
target with which to counter multidrug-resistant cancer (27). Therefore, the expression of STAT3 and its upstream gene, EGFR, was measured in doxorubicin-resistant human breast cancer MCF7/DOX cells, and the involvement of QIC1 in the MCF7/DOX cells was also investigated. It was found that STAT3 as well as its upstream gene, EGFR, were overexpressed in the MCF7/DOX cells (Fig. 5A-D). QIC1 may downregulate the level of the STAT3 and EGFR proteins (Fig. 5E) in MCF7/DOX cells. An MTT assay demonstrated that QIC1 inhibited MCF7/DOX cell proliferation in a dose-dependent manner, and that non-toxic (cell survival rate $>90 \%$ ) doses of QIC1 $(1 \mu \mathrm{M}, 2 \mu \mathrm{M})$ enhanced the anticancer activity of doxorubicin in the MCF7/DOX cells, consequently reversing the drug resistance of MCF7/DOX cells (Fig. 5F and G). Therefore, QIC1 may also reverse multi-drug resistance in tumor cells.

\section{Discussion}

Currently, chemotherapy remains an important therapeutic approach for the majority of types of cancer. However, this approach is not always fully effective, either due to an initial lack of response by the tumor, or as a result of the development of drug resistance over time. Thus, there is a continued need to develop alternative effective treatment agents. Previous studies have shown that quinolone derivatives and indolone derivatives exhibit anticancer activity in human cancer cell lines $(28,29)$. Therefore, the quinolone-indolone conjugate, QIC1, was synthesized by introducing indolin-2-one to the quinolone C-3 position. The present study focused on the effect of QIC1 on the EGFR pathway in cancer cells.

It was observed that QIC1 inhibited proliferation and induced apoptosis in HepG2, MCF7 and A549 cancer cell lines. The present results also demonstrated that QIC1 is a non-cytotoxic compound. The primary apoptotic signal transduction cascades generally converge onto a common pathway that regulates proteins involved in cell survival, such as Bcl-2, and in cell death, such as Bax. Reduced expression of Bax has been reported in breast cancer and is hypothesized to be responsible for the development of relative drug resistance $(30,31)$. In the present study, a dose-dependent increase in Bax level and a decrease in Bcl-2 expression was observed following treatment with QIC1, which suggested that QIC1 is likely to promote apoptosis.

The EGFR signaling pathway is required for the enhanced proliferation and survival of cancer cells. In the current study, the expression of EGFR in HepG2, MCF7 and A549 cancer cell lines was measured. The results demonstrated a prominent decrease in EGFR and phospho-EGFR level in all cell lines that were treated with QIC1. AKT and STAT3, which are downstream molecules of the EGFR pathway, are generally found to be constitutively active in the majority of cancers (32-35). The present study demonstrated reduced activity of these two molecules following treatment with QIC1. These results indicated that the inhibition of EGFR and its downstream molecules, may affect the viability of HepG2, MCF7 and A549 cells. HK2 is overexpressed and activated in a number of types of cancer, which facilitates the growth and proliferation of cancer cells. Decreased HK2 expression was observed in all cell lines exposed to QIC1. A previous study also found that HK2 may be regulated by STAT3. Therefore, the present study sought to further confirm whether QIC1 regulates cancer cell proliferation and apoptosis through the EGFR-STAT3-HK2 pathway. The current results showed that the anticancer activity of QIC1 is counteracted by the administration of STAT3 siRNA and 3-BrPA.

Additional experiments were designed to investigate the effects of QIC1 in doxorubicin-resistant MCF7/DOX human breast cancer cells. It was found that STAT3 as well as its upstream gene, EGFR, were overexpressed in MCF7/DOX cells, and that QIC1 downregulated the level of STAT3 and EGFR proteins. It was also demonstrated that QIC1 inhibited MCF7/DOX cell proliferation in a dose-dependent manner, and that non-toxic (cell survival rate $>90 \%$ ) doses of QIC1 enhanced the anticancer activity of doxorubicin in MCF/DOX cells. The present study provides evidence that QIC1 may reverse multidrug resistance in cancer cells.

In conclusion, QIC1 appears to be a promising novel anticancer agent, which exhibits high efficacy in cancer cells. It inhibits cancer cell growth by targeting the EGFR-STAT3-HK pathway, thus reversing multidrug resistance in cancer cells.

\section{Acknowledgements}

This study was supported by a grant from the Key Science and Technology Fund of Henan Province in China (grant no. 122102310558).

\section{References}

1. Shehata MA and Karim NA: Influenza vaccination in cancer patients undergoing systemic therapy. Clin Med Insights Oncol 8: 57-64, 2014.

2. Lo HW, Hsu SC and Hung MC: EGFR signaling pathway in breast cancers: From traditional signal transduction to direct nuclear translocalization. Breast Cancer Res Treat 95: 211-218, 2006.

3. Ribeiro FA, Noguti J, Oshima CT and Ribeiro DA: Effective targeting of the epidermal growth factor receptor (EGFR) for treating oral cancer: A promising approach. Anticancer Res 34: 1547-1552, 2014.

4. Zahonero C and Sánchez-Gómez P: EGFR-dependent mechanisms in glioblastoma: Towards a better therapeutic strategy. Cell Mol Life Sci 71: 3465-3488, 2014.

5. Arfaoui A, Kriaa L, Znaidi N, Gritli S, Bouacha H, Zermani R and Rammeh S: Over-expression of EGFR is closely correlated to poor prognosis in Tunisian patients with non-small cell lung adenocarcinoma. J Immunoassay Immunochem 35: 256-268, 2014.

6. Su JC, Lin KL, Chien CM, Chuang PW, Chang LS and Lin SR: Concomitant inactivation of the epidermal growth factor receptor, phosphatidylinositol 3-kinase/Akt and Janus tyrosine kinase 2/signal transducer and activator of transcription 3 signalling pathways in cardiotoxin III-treated A549 cells. Clin Exp Pharmacol Physiol 37: 833-840, 2010.

7. Subramaniam A, Shanmugam MK, Ong TH, Li F, Perumal E, et al: Emodin inhibits growth and induces apoptosis in an orthotopic hepatocellular carcinoma model by blocking activation of STAT3. Br J Pharmacol 170: 807-821, 2013.

8. Fang Z, Tang Y, Fang J, Zhou Z, Xing Z, et al: Simvastatin inhibits renal cancer cell growth and metastasis via AKT/mTOR, ERK and JAK2/STAT3 pathway. PLoS One 8: e62823, 2013.

9. Weerasinghe P, Garcia GE, Zhu Q, Yuan P, Feng L, et al: Inhibition of Stat 3 activation and tumor growth suppression of non-small cell lung cancer by G-quartet oligonucleotides. Int J Oncol 31: 129-136, 2007.

10. Zhang X, Yue P, Page BD, Li T, Zhao W, et al: Orally bioavailable small-molecule inhibitor of transcription factor Stat3 regresses human breast and lung cancer xenografts. Proc Natl Acad Sci USA 109: 9623-9628, 2012 
11. Kortylewski M and Yu H: Stat3 as a potential target for cancer immunotherapy. J Immunother 30: 131-139, 2007.

12. Tai WT, Cheng AL, Shiau CW, Huang HP, Huang JW, et al: Signal transducer and activator of transcription 3 is a major kinase-independent target of sorafenib in hepatocellular carcinoma. J Hepatol 55: 1041-1048, 2011.

13. Warburg O: On the origin of cancer cells. Science 123: 309-314, 1956.

14. Koppenol WH, Bounds PL and Dang CV: Otto Warburg's contributions to current concepts of cancer metabolism. Nat Rev Cancer 11: 325-337, 2011.

15. Hernández JF, Urueña CP, Cifuentes MC, Sandoval TA, Pombo LM, Castañeda D, Asea A and Fiorentino S: A Petiveria alliacea standardized fraction induces breast adenocarcinoma cell death by modulating glycolytic metabolism. J Ethnopharmacol 153: 641-649, 2014.

16. Chen WL, Wang JH, Zhao AH, Xu X, Wang YH, et al: A distinct glucose metabolism signature of acute myeloid leukemia with prognostic value. Blood 124: 1645-1654, 2014.

17. Venmar KT,Kimmel DW, Cliffel DE and Fingleton B: IL4 receptor $\alpha$ mediates enhanced glucose and glutamine metabolism to support breast cancer growth. Biochim Biophys Acta 1853: 1219-1228, 2015.

18. Chen J, Zhang S, Li Y, Tang Z and Kong W: Hexokinase 2 overexpression promotes the proliferation and survival of laryngeal squamous cell carcinoma. Tumour Biol 35: 3743-3753, 2014.

19. Wang TX, Liu YH and Shi XY: Oridonin induces apoptosis of HepG2 cells via downregulating STAT3-HK II pathway. Chinese Pharmacological Bulletin 30: 397-402: 2014 (In Chinese).

20. Li J, Liu T, Zhao L, Chen W, Hou H, et al: Ginsenoside 20(S)-Rg3 inhibits the Warburg effect through STAT3 pathways in ovarian cancer cells. Int J Oncol 2: 775-781, 2015.

21. Li Z, Li X, Wu S, Xue M and Chen W: Long non-coding RNA UCA1 promotes glycolysis by upregulating hexokinase 2 through the mTOR-STAT3/microRNA143 pathway. Cancer Sci 105: 951-955, 2014.

22. Moiseeva EP, Heukers R and Manson MM: EGFR and Src are involved in indole-3-carbinol-induced death and cell cycle arrest of human breast cancer cells. Carcinogenesis 28: 435-445, 2007.

23. Chen J, Wang W, Wang H, Liu X and Guo X: Combination treatment of ligustrazine piperazine derivate DLJ14 and adriamycin inhibits progression of resistant breast cancer through inhibition of the EGFR/PI3K/Akt survival pathway and induction of apoptosis. Drug Discov Ther 8: 33-41, 2014.

24. Yamaguchi $\mathrm{H}$ and Wang HG: The protein kinase PKB/Akt regulates cell survival and apoptosis by inhibiting Bax conformational change. Oncogene 20: 7779-7786, 2001.
25. Le Gall M, Chambard JC, Breittmayer JP, Grall D, Pouysségur J and Van Obberghan Schilling E: The p42/p44 MAP kinase pathway prevents apoptosis induced by anchorage and serum removal. Mol Biol Cell 11: 1103-1112, 2000.

26. Grandis JR, Drenning SD, Chakraborty A, Zhou MY, Zeng Q, et al: Requirement of Stat 3 but not Stat 1 activation for epidermal growth factor receptor-mediated cell growth in vitro. J Clin Invest 102: 1385-1392, 1998.

27. Tai WT, Cheng AL, Shiau CW, Liu CY, Ko CH, et al: Dovitinib induces apoptosis and overcomes sorafenib resistance in hepatocellular carcinoma through SHP-1-mediated inhibition of STAT3. Mol Cancer Ther 11: 452-463, 2012.

28. Wicke L, Engels JW, Gambari R and Saab AM: Synthesis and antiproliferative activity of quinolone nucleosides against the human myelogenous leukemia k-562 cell line. Arch Pharm (Weinheim) 346: 757-765, 2013.

29. Shou KJ, Li J, Jin Y and Lv YW: Design, synthesis, biological evaluation, and molecular docking studies of quinolone derivatives as potential antitumor topoisomerase I inhibitors. Chem Pharm Bull (Tokyo) 61: 631-636, 2013.

30. Bargou RC, Wagener C, Bommert K, Mapara MY, Daniel PT, et al: Overexpression of the death-promoting gene bax-alpha which is downregulated in breast cancer restores sensitivity to different apoptotic stimuli and reduces tumor growth in SCID mice. J Clin Invest 97: 2651-2659, 1996.

31. Bargou RC, Daniel PT, Mapara MY, Bommert K, Wagener C, et al: Expression of the bcl-2 gene family in normal and malignant breast tissue: Low bax-alpha expression in tumor cells correlates with resistance towards apoptosis. Int J Cancer 60: 854-859, 1995.

32. Blando JM, Carbajal S, Abel E, Beltran L, Conti C, et al: Cooperation between Stat 3 and Akt signaling leads to prostate tumor development in transgenic mice. Neoplasia 13: 254-265, 2011.

33. Lin J, Tang H, Jin X, Jia G and Hsieh JT: p53 regulates Stat3 phosphorylation and DNA binding activity in human prostate cancer cells expressing constitutively active Stat3. Oncogene 21: 3082-3088, 2002.

34. Vangala Jr, Dudem S, Jain N and Kalivendi SV: Regulation of PSMB5 protein and $\beta$ subunits of mammalian proteasome by constitutively activated signal transducer and activator of transcription 3 (STAT3): Potential role in bortezomib-mediated anticancer therapy. J Biol Chem 289: 12612-12622, 2014.

35. Wu K, Chang Q, Lu Y, Qiu P, Chen B, et al: Gefitinib resistance resulted from STAT3-mediated Akt activation in lung cancer cells. Oncotarget 4: 2430-2438, 2013. 